\title{
Dosage adjustment in medical patients with renal impairment at Groote Schuur Hospital
}

\author{
Eric Decloedt, Rory Leisegang, Marc Blockman, Karen Cohen
}

Background. Many drugs are eliminated by the kidneys and therefore may require dose adjustment in patients with renal impairment. The need for dose adjustment is frequently neglected by prescribers.

Methods. We reviewed folders of patients admitted to the Groote Schuur Hospital general medical wards between January and March 2008. Patients with renal impairment, defined as an estimated glomerular filtration rate (eGFR) $\leq 50 \mathrm{ml}$ per minute per $1.73 \mathrm{~m}^{2}$, were identified. In-patient prescriptions were captured if they were written after clinical notes indicated impaired renal function, or $\geq 1$ day after renal function tests were performed. We determined what proportion of these prescriptions required dose adjustment and whether drug doses were appropriately adjusted.

Results. We found renal impairment in 32\% (97/301) of medical admissions. There were 615 prescription entries for the 97 patients with renal impairment. Dose adjustment was required in $19 \%(117 / 615)$ of prescription entries, and only $32 \%(37 / 117)$ of these prescription entries were correctly dose adjusted. Of 97 patients, 69 received one or more drugs that required dose adjustment (median 1, range 1 - 5). All drug doses were correctly adjusted in $12 \%(8 / 69)$ of patients. Importantly, in the majority of patients $(59 \%(41 / 69))$ no doses had been correctly adjusted.

Conclusion. Consistent with international studies, drug dose adjustment in patients with renal impairment in a South African hospital was frequently neglected. Strategies to alert clinicians of the need for dose adjustment in renal impairment should be considered, including automated eGFR reporting and computerised aids to guide drug dosing, that account for renal impairment.

S Afr Med J 2010; 100: 304-306.
Failure to adjust dosing of renally excreted drugs in patients with renal impairment may lead to adverse effects, including nephrotoxicity. The incidence of in-hospital acute kidney injury attributed to drug nephrotoxicity is estimated at $19 \%{ }^{1}$ Druginduced nephrotoxicity and adverse effects can be reduced by adjusting the dosing of renally excreted drugs according to the patient's estimated glomerular filtration rate (eGFR), but this is often neglected by prescribers. In renally impaired patients, $29-74 \%$ of drug prescriptions requiring dose adjustment are not appropriately adjusted..$^{2-7}$

We conducted a retrospective cross-sectional study to determine the prevalence of incorrect dosing of renally eliminated drugs among general medical patients with renal impairment at Groote Schuur Hospital (GSH), Cape Town. We aimed to determine what proportion of drugs prescribed to patients with renal impairment (defined as an eGFR of $\leq 50 \mathrm{ml}$ per minute per $1.73 \mathrm{~m}^{2}$ ) in the general medical wards required dose adjustment; and to determine whether drug doses were appropriately adjusted in these patients, using Drug Prescribing in Renal Failure as our reference for dose adjustments. ${ }^{8}$

Division of Clinical Pharmacology, Department of Internal Medicine, University of Cape Town and Groote Schuur Hospital, Cape Town

Eric Decloedt, MB ChB, BSc Pharmacol (Hons)

Rory Leisegang, MB ChB, BSc Eng (Hons)

Marc Blockman, MB ChB, MMed (Clin Pharmacol), BPharm, Dip Int Res

Ethics

Karen Cohen, MB ChB, FCFP (SA), MSc (Epid), Dip HIV Man, Dip Obst

\section{Methods}

We reviewed folders of all patients admitted to the general medical wards of GSH between 1 January and 31 March 2008 The serum creatinine concentration, measured on the day of admission to the medical ward, was extracted. If this had not been measured at that time, the creatinine result with which the patient was referred to the medical ward from casualty or a referring hospital was used. eGFR was calculated using the abbreviated Modification of Diet in Renal Disease study equation (MDRD): eGFR=186 $\times(\mathrm{Scr} / 88.4)^{-1.154} \times(\text { age })^{-0.203}$ $\times(0.742$ if female $) \times(1.212$ if African-American $){ }^{9-11}$ This formula requires age, sex, creatinine and ethnicity as inputs. As recommended by the South African Renal Society, the adjustment factor recommended for African-American patients was included in the equation for black patients, and omitted in those of other ethnicity. ${ }^{11}$ We categorised patient ethnicity as black if the patient was recorded as Xhosa-speaking in the admission record. We included all patients with renal impairment, defined as an eGFR $\leq 50 \mathrm{ml}$ per minute per 1.73 $\mathrm{m}^{2}$, who received treatment in the medical ward for at least 1 day after a serum creatinine test was performed. Drug details were extracted from prescription charts on the day that the clinical notes indicated that the renal function was reviewed. When no mention of the renal function was made in the clinical notes, we extracted prescribed drugs from prescription charts at least 1 day after the serum creatinine was measured. This step ensured that prescribers had had a 1-day period after renal function was assessed in which to adjust drug doses. We used Drug Prescribing in Renal Failure to identify drugs requiring dose adjustment, and to determine if the appropriate dose adjustment for the patient's eGFR had occurred. ${ }^{8}$ 


\section{Data management and analysis}

Data from the patient folders were entered into an AccessTM database. Statistical analyses were performed using Stata 10.0 (Stata Corporation, College Station, Texas, USA). Numerical variables were summarised using median and range or interquartile range (IQR). Associations between categorical variables were explored using the chi-square test of association.

\section{Ethical approval}

Ethical approval was obtained from the Human Research Ethics Committee of the Faculty of Health Sciences, University of Cape Town.

\section{Results}

Between January and March 2008, 433 patients were admitted to the general medical wards. We located and assessed 317 folders, of which 303 had complete notes. The eGFR was $>50 \mathrm{ml}$ per min per $1.73 \mathrm{~m}^{2}$ in 204 patients, and 2 patients with renal impairment were transferred from the ward to the intensive care unit within 24 hours of admission. Therefore, 97 patients fulfilled our inclusion criteria and were included in the final analysis: 32\% (97/301) of medical admissions.

The median age of patients with renal impairment was 46 years; $52 \%$ were female; the median number of drugs prescribed per patient was 6 (range 1 - 15) and 55\% (53/97) had $\geq 5$ drugs prescribed. The median eGFR was $19 \mathrm{ml}$ per min per $1.73 \mathrm{~m}^{2}$ (IQR 9 - 33), with a median creatinine of $448 \mu \mathrm{mol} / 1$ (IQR 314 - 950) (Table I).

There were 615 prescription entries for the 97 patients with renal impairment. In total, 110 different drugs were prescribed, and some prescribed on multiple occasions. Dose adjustment was required in $19 \%(117 / 615)$ of prescription entries. Of the 117 prescription entries, only 37 were correctly dose adjusted $(32 \%)$.

Co-amoxiclavulanic acid was the most frequently prescribed drug that required dose adjustment, which was done in $21 \%$ $(5 / 24)$ of cases; followed by atenolol and gliclazide, adjusted in $26 \%(5 / 19)$ of cases and $25 \%(2 / 8)$ of cases respectively. Of the most frequently prescribed drugs, ethambutol was the only drug correctly dose adjusted in all cases (6/6). Ciprofloxacin and digoxin both required dose adjustment in 5 cases, and remained unadjusted in all cases.

Of the 97 patients, $71 \%(69 / 97)$ received $\geq 1$ drug that required dose adjustment (median 1; range 1 - 5). Only $12 \%$

\begin{tabular}{ll}
\multicolumn{2}{l}{ Table I. Patient characteristics $(\mathbf{N = 9 7 )}$} \\
\hline Sex & $50(52 \%)$ female \\
Ethnicity & $33(34 \%)$ black \\
Median age (IQR) & $46(23-79)$ years \\
Median weight (IQR) & $64(54-73) \mathrm{kg}^{*}$ \\
Median creatinine (IQR) & $448(314-950) \mu \mathrm{mol} / \mathrm{l}$ \\
Median GFR (IQR) & $19.2(8.9-33.1) \mathrm{ml} / \mathrm{min} / 1.73 \mathrm{~m}^{2}$ \\
Median drugs & $6(1-15)$ \\
prescribed (range) & \\
$\geq 5$ drugs per script & $53 / 97(55 \%)$ \\
*Weight available for only $58 / 97(60 \%)$ patients. \\
\hline
\end{tabular}

$(8 / 69)$ of patients had all of their drugs correctly adjusted; $29 \%$ $(20 / 69)$ of patients had some drugs correctly adjusted; $59 \%$ $(41 / 69)$ of patients had no drugs correctly adjusted.

On univariate analysis, we found no association between dose adjustment and sex, age, extent of renal impairment or the number of prescription items. Nine patients were documented to have received dialysis before or on the day when the prescription was reviewed for dose adjustment. No patients receiving dialysis had all of their prescription items correctly adjusted, and $89 \%(8 / 9)$ had one or more inappropriately dosed prescription entries.

\section{Discussion}

Renal impairment occurred in $32 \%(97 / 301)$ of patients admitted for $\geq 24$ hours in the general medical wards at GSH. Dose adjustment was required in 19\% of prescription entries in patients with renal impairment, and only $32 \%$ of those prescriptions were correctly dose adjusted. One or more drugs requiring dose adjustment were prescribed to $71 \%(69 / 97)$ of patients with renal impairment. In most patients prescribed drugs requiring dose adjustment (59\%), none was correctly adjusted. Worryingly, 8/9 dialysed patients had at least one prescription item incorrectly dosed.

To our knowledge, this is the first study in general medical patients with renal impairment of the frequency of dose adjustment of renally excreted drugs in South Africa. Our findings are in keeping with international studies, which found inappropriate dose adjustment in $29-74 \%$ of patients with renal impairment. ${ }^{2-7}$

Failure to adjust drug doses has several possible explanations. Prescribers might not review renal function results before prescribing. Reference texts to guide adjustment in renal impairment might not be freely available in the hospital wards. Prescribers who do not routinely check whether dose adjustment is required in patients with renal impairment might selectively adjust drugs that are wellknown to require dose adjustment, and be unaware of the need for dose adjustment of other drugs. Prescribers might have used different methods from ours to calculate and define renal impairment, such as only using serum creatinine as an indicator of renal impairment rather than calculating the eGFR. This omission might have resulted in significant renal impairment being missed in lighter patients. The hospital laboratory does not routinely calculate creatinine clearances when a creatinine test is requested, and clinicians would therefore have to calculate the clearance themselves before prescribing. When calculating eGFR, prescribers might have used the Cockroft-Gault formula (eGFR=[(140-age) $\times$ weight $] /$ Scr $\times 0.85$ if female) rather than the MDRD formula. ${ }^{10}$ The Cockroft-Gault formula is unlikely to have been used to calculate eGFR in many of the patients in our study, because weight is a variable required when using the Cockroft-Gault formula, and weight was only documented in $60 \%$ of patients with renal impairment. Lastly, a critically ill patient may experience rapid changes in renal function, making eGFR extremely difficult and unreliable. The limitations of currently available eGFR equations have been widely acknowledged 
in patients with acute renal impairment. ${ }^{12,13}$ Prescribers might have opted not to dose adjust in patients with acute deterioration in renal function, with the expectation that renal function would improve and out of concern for under-dosing.

Our study has limitations. It was conducted retrospectively so as not to influence prescribing habits. Retrospective data collection may be hampered by missing and incomplete data, and we could not locate folders in $30 \%$ of medical admissions, which decreased our sample size. However, the patients with missing folders are unlikely to have differed in any systematic way from patients whose folders were located. It is therefore unlikely that inability to locate their folders introduced bias. As the study was retrospective, we could not determine what method, if any, prescribers used to identify renal impairment and assess the severity of renal impairment in patients in our study. We used the internationally recommended MDRD formula, which includes an adjustment factor in the equation for African-Americans to estimate GFR. ${ }^{10}$ The South African Renal Society recommends use of this adjustment factor in black African patients, and omission of the adjustment factor in patients of all other ethnic backgrounds. ${ }^{11}$ As this was a retrospective chart review, we could not acquire self-defined ethnicity data, or data on ethnic ancestry, from patients. We therefore identified black African patients as those who were recorded as Xhosa-speaking on their admission records, which might have resulted in misclassification of some patients. To our knowledge, there are no data on performance of the MDRD formula in a southern African population, and there may be differences between our population and the population in which the formula was originally developed and validated. Dose adjustment in rapidly changing renal function is controversial, and some of the failure to dose adjust might have been due to prescribers electing not to adjust dosing during acute renal impairment. Our study design did not allow us to differentiate between acute and chronic renal impairment. Lastly, prescribers might have used a different reference source from that which we used.

Studies have suggested methods to improve adherence to dose adjustment recommendations in renal impairment. Computer-guided drug dosing for inpatients with renal impairment has been shown to result in an increase in correct dosing of renally eliminated drugs..$^{20}$ Automated eGFR reporting in renal function reports are already standard practice in many South African private laboratories and may alert the prescribing clinician to the presence of significant renal impairment.

We found that clinicians frequently neglected to adjust doses in renally impaired patients. Renal impairment is common in medical inpatients, and it is therefore important to increase awareness of the importance of dose adjustment and improve adherence to dose adjustment recommendations. Increasing availability of reference texts in medical wards, providing clinicians with an eGFR with all creatinine results, and electronic aids to identify drugs requiring dose adjustment, should be considered.

We thank Professor Charles Swanepoel for his input.

References

1. Uchino S, Kellum JA, Bellomo R, et al. Acute renal failure in critically ill patients: a multinational, multicenter study. JAMA 2005; 294(7): 813-818.

2. Cantu TG, Ellerbeck EF, Yun SW, Castine SD, Kornhauser DM. Drug prescribing for patients Cantu TG, Ellerbeck EF, Yun SW, Castine SD, Kornhauser DM. Drug p
with changing renal function. Am J Hosp Pharm 1992; 49(12): 2944-2948.

3. Salomon L, Deray G, Jaudon MC, et al. Medication misuse in hospitalized patients with renal impairment. Int J Qual Health Care 2003; 15(4): 331-335.

4. Pillans PI, Landsberg PG, Fleming AM, Fanning M, Sturtevant JM. Evaluation of dosage adjustment in patients with renal impairment. Intern Med J 2003; 33(1-2): 10-13.

5. van Dijk EA, Drabbe NR, Kruijtbosch M, De Smet PA. Drug dosage adjustments according to renal function at hospital discharge. Ann Pharmacother 2006; 40(7-8): 1254-1260.

6. Wong NA, Jones HW. An analysis of discharge drug prescribing amongst elderly patients with renal impairment. Postgrad Med J 1998; 74(873): 420-422.

7. Sweileh WM, Janem SA, Sawalha AF, et al. Medication dosing errors in hospitalized patients Sweileh WM, Janem SA, Sawalha AF, et al. Medication dosing errors in hospitalized patients
with renal impairment: a study in Palestine. Pharmacoepidemiol Drug Saf 2007; 16(8): 908-912. 8. Aronoff GR, Bennett WM, Berns JS, et al. Drug Prescribing in Renal Failure: Dosing Guidelines for Adults and Children. 5th ed. Philadelphia: American College of Physicians, 2007.

9. Levey AS, Coresh J, Greene T, et al. Using standardized serum creatinine values in the modification of diet in renal disease study equation for estimating glomerular filtration rate. Ann Intern Med 2006; 145(4): 247-254.

10. National Kidney Foundation. KDOQI Clinical Practice Guidelines for Chronic Kidney Disease: Evaluation, Classification, and Stratification. 2002. http://www.kidney.org/PROFESSIONALS/ kdoqi/guidelines_ckd/p5_lab_g4.htm (accessed 30 June 2008).

11. South African Renal Society. Guidelines for the Optimal Care of Patients on Chronic Dialysis in South African Renal Society. Guidelines for the Optimal Care of Patients on Chronic Dialysis
South Africa. 2006. www.kznhealth.gov.za/medicine/sars.pdf (accessed 30 June 2009).

12. DeBellis RJ, Smith BS, Cawley PA, Burniske GM. Drug dosing in critically ill patients with renal failure: a pharmacokinetic approach. J Intensive Care Med 2000; 15(6): 273-313.

13. Lameire N, Hoste E. Reflections on the definition, classification, and diagnostic evaluation of acute renal failure. Curr Opin Crit Care 2004; 10(6): 468-475.

14. Chertow GM, Lee J, Kuperman GJ, et al. Guided medication dosing for inpatients with renal insufficiency. JAMA 2001; 286(22): 2839-2844.

Accepted 14 January 2010. 